\title{
Targeting Homeostatic T Cell Proliferation to Control Beta-Cell Autoimmunity
}

\author{
Debora Vignali ${ }^{1} \cdot$ Paolo Monti $^{1}$
}

Published online: 16 March 2016

(C) The Author(s) 2016. This article is published with open access at Springerlink.com

\begin{abstract}
Immunomodulation of the autoreactive T cell response is considered a major strategy to control beta-cell autoimmunity, both in the natural history of type 1 diabetes and in islet transplantation, which can be affected by autoimmunity recurrence. So far, these strategies have had modest results, prompting efforts to define novel cellular and molecular targets to control autoreactive $\mathrm{T}$ cell expansion and activation. Novel findings highlighted the important role of the homeostatic cytokine interleukin-7 in inducing proliferation and differentiation of autoreactive $\mathrm{T}$ cell clones that causes beta-cell autoimmunity. In this review, we discuss recent evidences and novel findings on the role of IL-7 mediated homeostatic T cell proliferation in the process of beta-cell destruction and evidences of how targeting IL-7 and its receptor could be an innovative and effective strategy to control beta-cell autoimmunity.
\end{abstract}

Keywords Autoreactive T cells $\cdot$ Homeostatic proliferation · Interleukin- $7 \cdot$ Type 1 diabetes $\cdot$ Islet transplantation

This article is part of the Topical Collection on Immunology and Transplantation

Paolo Monti

paolo.monti@hsr.it

Debora Vignali

debora.vignali@hsr.it

1 Transplant Immunology Unit, Diabetes Research Institute (DRI), IRCCS San Raffaele Scientific Institute, Via Olgettina 60, 20132 Milan, Italy

\section{Introduction}

Type 1 diabetes is caused by the selective destruction of pancreatic insulin-producing beta-cells by an immunemediated reaction, predominantly mediated by autoreactive T cells [1]. CD4+ and CD8+ T cells able to recognize MHC class II and class I restricted epitopes of the beta-cellassociated antigens glutamic acid decarboxilase 65 (GAD65), proinsulin, islet tyrosine phosphatase (IA-2), and zinc transporter 8 [2] have been found in patients with type 1 diabetes using proliferation assays, ELISPOT, and fluorescent class I and class II MHC multimers [3]. These studies have also highlighted several key observations concerning the nature of the $\mathrm{T}$ cell response toward betacells. First, while naive $T$ cells specific for beta-cell antigens are commonly found in subjects with no signs of betacell autoimmunity [4•], in patients with type 1 diabetes autoreactive $T$ cells show signs of previous antigen encounter, such as telomere shortening [5•], activation in the absence of co-stimulatory signals [6], and the expression of the memory marker CD45RO [5•]. Second, an autoreactive memory $\mathrm{T}$ cell response is difficult to control with standard immunosuppression and is highly refractory to immunomodulatory molecules. This is testified by the limited efficacy of recent clinical trials aiming to prevent or delay immune-mediated beta-cell loss using GAD65 vaccination [7], CTLA4-Ig [8], and humanized anti-CD3 antibody [9]. More aggressive approaches based on profound $\mathrm{T}$ cell depletion, although effective early after treatment [10], were later affected by frequent relapse of the autoimmune response $[11,12]$. Third, generation and expansion of autoreactive $\mathrm{T}$ cell clones can occur under the influence of homeostatic proliferation mediated by interleukin-7 (IL-7) [13]. The canonical antigen-specific proliferation pathway relies on the autocrine production of IL-2 and 
the upregulation of the IL-2 receptor alpha (also known as CD25). Therefore an important class of immunomodulatory molecules was developed to target this pathway including calcineurin inhibitors and anti-CD25 antibodies. Recent evidences however clearly showed that $\mathrm{T}$ cells can proliferate and acquire a memory phenotype upon activation of the IL-7/IL-7R axis [14••]. Moreover, recent findings suggest that IL-7 is involved in the generation of T cells with a stem cell-like memory phenotype (memory stem $\mathrm{T}$ cells, Tscm) [15••] and in the reprogramming of $\mathrm{T}$ cell bio-energetic metabolism for $\mathrm{T}$ cell proliferation [16]. While the interest on the homeostatic $\mathrm{T}$ cell proliferation pathway is increasing in autoimmunity, there is a substantial lack of molecules targeting this pathway in humans and trials to assess whether this could be an effective approach to control $\mathrm{T}$ cell-mediated beta-cell autoimmunity. In this article, we discuss how the IL-7/IL-7R pathway can contribute to the development of type 1 diabetes and how preclinical models have demonstrated the efficacy of a selective targeting of this pathway. Finally, we discuss how, and in which clinical setting, the targeting of the IL-7/IL-7R pathway can be a therapeutic option for the prevention and treatment of beta-cell autoimmunity.

\section{IL-7 Production and Regulation}

IL-7 is secreted by stromal cells located in the bone marrow, in peripheral lymphoid organs, and in the gastrointestinal tract [17]. These cells have yet to be fully characterized; however, it appears that IL-7 transcripts are produced at a constitutive level and are not influenced by extrinsic stimuli such as the concentration of IL-7 in a negative feedback or the size of the T cell compartment. Peripheral IL-7 concentration relies on consumption by IL7 receptor positive T cells. IL-7 production at a fixed rate and consumption keeps the serum concentration of IL-7 below $5 \mathrm{pg} / \mathrm{ml}$ in physiological conditions. [18] This represents a limiting factor for $\mathrm{T}$ cell expansion. In an immune system with a full $\mathrm{T}$ cell compartment, the concentration of IL-7 is sufficient for the survival of a finite number of T cells. IL-7 also maintains a slow rate of T cell proliferation to counterbalance physiological $\mathrm{T}$ cell death. When the peripheral $\mathrm{T}$ cell number is reduced (lymphopenia), the serum concentration of IL-7 rises to supraphysiological levels (Fig. 1). Remaining T cells in a lymphopenic environment and in an IL-7 rich milieu enter the cell cycle, and homeostatic $\mathrm{T}$ cell proliferation induced by IL-7 occurs. The process is self-limiting as restoring the number of peripheral $\mathrm{T}$ cells corresponds to an increased consumption of IL-7. Homeostatic T cell proliferation stops when the physiological $\mathrm{T}$ cell number and IL-7 concentration are fully restored.

\section{The IL-7/IL-7 Receptor Signaling Pathway}

The high affinity IL-7 receptor results from the IL-7-mediated crosslinking of the extracellular domains of the IL-7 receptor alpha-chain (IL-7R $\alpha$; also known as CD127) and the common cytokine receptor- $\gamma$ chain $\left(\gamma_{\mathrm{c}}\right.$, also known as CD132). Tyrosine kinases Janus kinases (JAK1) and JAK3 which are linked to the intracellular domain of the $\gamma_{c}$ mutually phosphorylate and their increased kinase activity, thus inducing phosphorylation of the IL-7R $\alpha$ intracellular domain and allowing the binding of the signal inducer transducer and activator of transcription factor 5 (STAT-5) which dimerizes and translocates to the nucleus activating gene transcription [19]. Once in the nucleus, STAT5 regulates the expression of many targets genes involved in $\mathrm{T}$ cell proliferation and survival, including the upregulation of prosurvival Bcl-2 family members, such as the main targets BCL-2, MCL-1, and BC-XL [20] and downregulation of proapoptotic members of the BCL-2 family, such as BAX or BIM [21]. Other STAT5regulated genes are Runt-related transcription factor 3 (Runx3), essential for T cell development and differentiation, and the suppressor of cytokines signaling (SOCS), necessary for negative feedback regulation of the cytokine signaling and $T$ cell compartment homeostasis.

In addition to survival, the IL-7R signaling induces $\mathrm{T}$ cell proliferation through the repression of the cell cycle inhibitor p2 $7^{\text {kip } 1}$ [22] and preventing degradation of Cdc25A [23]. T cell proliferation is an energy-demanding process and necessitates the incorporation of nutrient to increase the biomass needed to produce daughter cells [24]. In order to meet the bio-energetic demands for proliferation, $\mathrm{T}$ cells activate aerobic glycolysis. Despite a lower efficiency in ATP production as compared to oxidative phosphorylation, glycolysis is an anabolic process and provides also fundamental nutrients for the synthesis of biological macromolecules [25]. The IL-7 signaling activates the PI3K/Akt pathway, which is fundamental for $\mathrm{T}$ cell proliferation. To sustain the bio-energetic and metabolic needs, IL-7R signaling is also involved in the activation of the glycolytic machinery, including the Aktmediated upregulation of the glucose transporter 1 (GLUT1) [26] on the cell surface and the upregulation of the glycolytic enzymes hexokinase II (HXKII) [16]. Excision of IL-7R on mature $T$ cells in vivo results in size and growth reduction of $T$ cell compartment due to homeostatic mechanism impairment and metabolic cellular machinery liability [27].

\section{IL-7 in Beta-Cell Autoimmunity}

In order to protect an individual from a wide range of different pathogens, the $\mathrm{T}$ cell compartment needs to guarantee a broad diversity of antigen specificity and a sufficient cell number to mount an effective immune response [28]. The $\mathrm{T}$ cell 
Fig. 1 IL-7-mediated homeostatic $\mathrm{T}$ cell proliferation. In normal conditions, stromal cells constitutively produce IL-7 which is consumed by T cells and kept at low levels in the periphery. After T cell depletion, IL-7 concentration becomes elevated and remaining $\mathrm{T}$ cell start to proliferate in the IL-7 rich milieu. During expansion, autoreactive $\mathrm{T}$ cell clones can undergo expansion and are included in a higher number in the reconstituted $\mathrm{T}$ cell pool

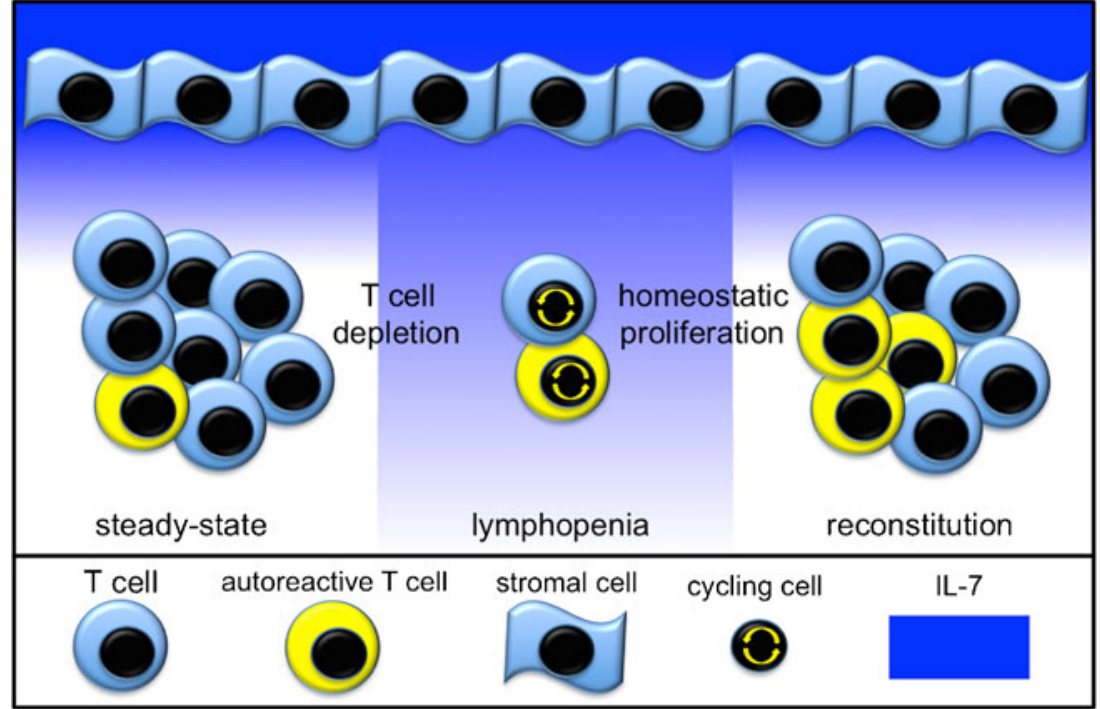

repertoire can also include $\mathrm{T}$ cell clones reactive to self-antigens, which escaped the negative selection process in the thymus and circulate as naive $\mathrm{T}$ cells. These potentially pathogenic clones are prevented from activation and proliferation mainly by their low affinity for the cognate MHC-peptide antigen complex which can deliver only weak signals as compared to high affinity T cell receptors [29•]. The TCR signal strength determines the T cell capacity to produce IL-2 and to upregulate the IL-2R $\alpha$ (also known as CD25) for an effective expansion and differentiation in a $\mathrm{T}$ cell compartment with a self-limiting size [30].

This competitive advantage of $\mathrm{T}$ cells with a high affinity TCR is lost in an IL-7 rich milieu. While the IL-2R $\alpha$ has an inducible expression regulated by $T$ cell activation through the TCR, the IL-7R $\alpha$ is constitutively expressed by all T cells and can induce $\mathrm{T}$ cell proliferation when the circulating concentrations of IL-7 reach supra-physiological levels [28]. Moreover, the low affinity TCR-MHC self-peptide interactions, which are required for $\mathrm{T}$ cell persistence, can give a selective advantage to autoreactive T cell clones [29•]. Perturbations of the IL-7/IL-7R axis can be associated with the activation of autoreactive T cells.

In animal models, the non-obese diabetic (NOD) mouse was shown to be affected by a chronic state of lymphopenia, which is permissive for autoreactive homeostatic T cell expansion under the influence of IL-21 [31]. Increasing the number of circulating T cells by injection of complete Freund adjuvant (CFA) at 3 weeks of age efficiently reduced the incidence of diabetes. Direct evidences of the role of IL-7 in the autoimmune beta-cell destruction process come from the rat insulin promoter (RIP)-lymphocytic choriomeningitis virus glycoprotein (GP)-transgenic mouse model [32•]. In this model, adoptive transfer of Smarta T cells restricted for the I-Ab-restricted LCMV GP61-80 (p13) epitope together with polyclonal CD8 T cells did not modify the incidence of diabetes. An increase in the incidence of diabetes was reported only when lymphopenia was induced with cyclophosphamide or by exogenous administration of IL-7. The diabetogenic effect of cyclophosphamide or IL-7 injection can be neutralized by blocking the IL-7R with a monoclonal antibody.

Lymphopenia, which is associated with increased circulating levels of IL-7 represents a typical condition in which autoreactive $\mathrm{T}$ cells can expand [28]. A relatively high risk of autoimmunity development has been observed in patients undergoing bone marrow transplantation after conditioning regimen and profound $\mathrm{T}$ cell depletion. Immunosuppression in patients receiving islet allotransplantation was associated with IL-7-mediated expansion of autoreactive T cell clones specific for GAD65 [14••]. Patients at risk for or with diagnosed type 1 diabetes have normal circulating levels of IL-7. However, single nucleotide polymorphisms (SNPs) of the IL$7 \mathrm{R} \alpha$ were associated with an increased risk of developing type 1 diabetes and multiple sclerosis [33]. As for many other cytokine receptors, a soluble form of the IL-7R $\alpha$ has been identified and characterized for its capacity to bind to and inhibit the interaction of IL-7 with the surface receptor in T cells. In conditions of high blood glucose levels, the soluble IL-7R $\alpha$ undergoes a non-enzymatic glycation process, losing its buffering capacity of IL-7 biological activity [34]. High levels of circulating IL-7 can be found in neonates and persist for the first 6 months of life [35]. Both GAD65 and proinsulinspecific $\mathrm{T}$ cells with a naive phenotype are already present at birth, and IL-7 was shown to promote in vitro proliferation and activation of GAD65 and proinsulin-specific T cells obtained from cord blood samples.

An IL-7 rich milieu can affect immune tolerance to selfantigens by acting directly on the CD4+CD25+ regulatory $\mathrm{T}$ cells (Treg) [36•]. The Treg network is essential to limit proliferation of autoreactive T cell clones. Despite a low expression of the IL-7R $\alpha$, which makes Treg unresponsive to 
physiological concentrations of circulating IL-7, STAT5 phosphorylation can be detected at pathological circulating concentrations of IL-7. The Treg response to IL-7 appeared to correlate with the phenotype. While $\mathrm{CD} 45 \mathrm{RO}+$ memory Treg remain anergic, CD45RO naive Treg strongly proliferate in response to IL-7, thus acquiring a CD45RO+ memory phenotype. Importantly, once exposed to IL-7, both memory and naive Treg have a reduced capacity to inhibit proliferation of conventional $\mathrm{T}$ cells in response to autoantigens. The suppressive capacity is fully restored upon IL-7 withdrawal. This effect can be of importance in the islet transplantation setting in which prolonged exposure of Treg to high circulating concentrations of IL-7 can impair the Treg capacity to control the expansion of autoreactive and alloreactive T cells.

\section{IL-7 and the Generation of Memory Stem T Cells}

The memory T cell compartment is composed by several subsets in different stages of differentiation [30]. Conventionally, memory $\mathrm{T}$ cell populations can be divided according to the differential expression of CD45RA, CCR7, and CD62L into central memory (Tcm), effector-memory (Tem), and terminally differentiated effector-memory RA (Temra) [37]. All memory subsets are generated from a common naive T cell (Tn) precursor according to a progressive differentiation model [38]. In this model, Tn progress along a differentiation pathway in the order of Tn, Tcm, Tem, and Temra. The final step of differentiation generates terminally differentiated effector $\mathrm{T}$ cells that are short lived and undergo to massive apoptosis (contraction) when the immune response exhausts. It was unclear whether long-term $\mathrm{T}$ cell memory is maintained by the conventional memory $\mathrm{T}$ cell subsets or by a long-lived $\mathrm{T}$ cell precursor with self-renewal potential. In humans, conventional memory $\mathrm{T}$ cell subsets have indeed a rapid turnover and a half-life of few weeks $[39,40]$. The existence of a memory precursor with stem cell-like properties was first hypothesized

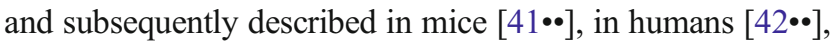
and in non-human primates [42••]. Tscm show a CD45RA+ $\mathrm{CCR} 7+$ naive phenotype associated with the expression of the memory markers IL-2Rbeta and CD95 [42••]. Generation of Tscm from naive $T$ cells involves the homeostatic cytokine IL7. In the classical antigen-specific activation pathway, $\mathrm{T}$ cell receptor engagement and IL-2 provides strong signals for $\mathrm{T}$ cell differentiation toward short-lived effector cells. In contrast, the homeostatic cytokine IL-7 sustains T cell proliferation without the robust differentiating activity of IL-2. In vitro priming of naive T cells in the presence of IL-7 results in the generation of $\mathrm{T}$ cells with phenotypic, functional, and gene expression attributes found in naturally arising Tscm cells [15••].

This is relevant to beta-cell autoimmunity because $\mathrm{T}$ cell memory to beta-cell antigens is established before the onset of type 1 diabetes and is then maintained for decades after the disease onset, as testified in patients with type 1 diabetes receiving islet or pancreas transplants, in which despite immunosuppression, can be associated with reactivation of autoimmunity [43, 44]. Given the role of the IL-7/IL-7R axis in T cell autoimmunity and the long lasting memory to beta-cell antigens observed in patients with type 1 diabetes, we previously hypothesized that a $\mathrm{T}$ cell precursor with stem cell-like properties could be generated by autoantigen stimulation in the presence of IL-7 [45]. Even though the existence of memory stem $T$ cells reactive to beta-cell antigens has to be proven, this population would be an ideal target cell for an innovative approach to beta-cell autoimmunity. Most strategies were designed to target activation/proliferation of pathogenic effector T cells. However, the identification and targeting of $\mathrm{T}$ cells that preserve long-term memory to beta-cell antigen would be able to eradicate the pathogenic $\mathrm{T}$ cell progeny. This approach is similar in principle to that hypothesized to treat cancer by targeting cancer stem cells [46]. In this theory, a permanent eradication of a tumor mass can be achieved by selective targeting of rare and slowly proliferating cancer stem cell precursors. In contrast, standard radio-chemotherapy targeting the vast majority of rapidly proliferating cancer cells but not cancer stem cells results in a high rate of tumor relapse.

\section{Targeting IL-7 Mediated Homeostatic T Cell Proliferation in Preclinical Models}

Molecules with the ability to directly or indirectly target the IL-7/IL-7R axis have been discovered and first tested in preclinical models (Table 1). The IL-7/IL-7R axis has been implicated in the development of autoimmune diabetes in the NOD mouse model. Two reports showed how IL-7R $\alpha$ blockade was effective to prevent and revert diabetes. In the first report $[47 \cdot \bullet]$, the selective inhibition of the IL-7R $\alpha$ using a monoclonal antibody was able to prevent diabetes after only 2-3 injections starting at week 9 of age. Disease remission was complete and durable, after a 4-week cycle of anti-IL$7 \mathrm{R} \alpha$ antibody starting at the time of diabetes onset. Anti-IL$7 \mathrm{R}$ antibody was reported to act by inhibiting diabetogenic effector-memory T cells (Tem). Diabetogenic Tem cells were not depleted by IL-7R $\alpha$ treatment but were instead reprogrammed to suppress IFN- $\gamma$ secretion and to upregulate the inhibitory receptor programmed death 1 (PD-1). Tem cells from anti-IL-7R $\alpha$-treated mice were also adoptively transferred in recipient mice without causing diabetes and demonstrating that IL-7-signaling deprivation was related to a state of cell intrinsic tolerance. In the second report [48••], treatment with the IL-7R $\alpha$ antibody resulted in the reduction of IFN- $\gamma$-producing CD4+ (TH1) and IFN- $\gamma$-producing CD8+ (Tc1) T cells. As shown also in the first report, IL-7R $\alpha$ antibody treatment induced upregulation of PD-1 expression in 
Table 1 Molecules targeting the IL-7/IL-7R axis

\begin{tabular}{lllll}
\hline Study phase & Molecule & IL-7/IL-7R specificity & Setting & Refs \\
\hline Clinical & Anti-IL-7R $\alpha$ mAb & Yes & T1D, MS & N/A \\
& Mycophenolate mofetil & No & Islet tx in T1D & {$[14 \bullet \bullet]$} \\
& JAK inhibitors & No & RA & {$[55]$} \\
Preclinical & Anti-IL-7R $\alpha$ mAb & Yes & Diabetes in NOD & {$[47 \bullet \bullet, 48 \bullet \bullet, 49 \bullet]$} \\
& & & Islet tx & \\
& Bz-423 & No & GVHD & {$[59 \bullet]$} \\
& 2-DG+ metformin & No & Lupus & {$[60 \bullet]$} \\
& SCD127 & Yes & Human & {$[34]$} \\
& Tat protein & Yes & Human & {$[54]$} \\
\hline
\end{tabular}

effector $\mathrm{T}$ cells that appeared to be necessary for maintenance of tolerance, which could be reversed by PD-1 blockade. Interestingly, in both models, IL-7R $\alpha$ antibody treatment was associated with an increased frequency of regulatory $\mathrm{T}$ cells. The same monoclonal antibody raised against the IL$7 \mathrm{R} \alpha$ was also tested on BALB/c mice receiving $\mathrm{C} 57 \mathrm{BL} / 6$ islets under the kidney capsule, as model of islet transplantation in streptozotocin-induced diabetes [49•]. When started 3 weeks before islet infusion, the anti-IL-7R $\alpha$ treatment induced indefinite pancreatic islet allograft survival. This is of interest in the field of islet transplantation where a single treatment with an anti-IL-7R $\alpha$ can potentially control both the autoimmune and the alloimmune response to islet allografts.

Biological and pharmacological regulators of the IL-7/IL$7 \mathrm{R}$ axis with a potential translation into the clinic have been identified. As for other cytokine receptors, a soluble form of the IL-7R $\alpha$ (sCD127) has been identified in humans [50]. sCD127 is generated both by alternative splicing or shedding of the membrane bound IL-7R $\alpha$. The amount of circulating sCD127 is regulated by single nucleotide polymorphism (SNP) of the IL-7R $\alpha$ gene [51]. A SNP found in exon 6 (rs6897932) determines the extent of exon splicing. Transcripts that skip exon 6 (C allele of rs6897932) encode sCD127 [52]. The polymorphism determines an increase of circulating levels of sCD127 and has been associated with an increased susceptibility to type 1 diabetes. SCD127 can bind to and inhibit IL-7 and is the only known endogenous regulator of the IL-7 biological activity [34]. sCD127 display a low affinity $(\mathrm{Kd}=10-8 \mathrm{M})$ for IL-7 which is 3 logs lower than the affinity of IL-7 for the membrane IL-7R $\alpha-\gamma_{\mathrm{c}}$-chain [53]. Nevertheless, sCD127 concentration in serum $(70-80 \mathrm{ng} / \mathrm{mL})$ is 4 logs higher than physiological IL-7 concentration IL-7 $(5 \mathrm{pg} / \mathrm{ml})$ and is therefore expected to significantly influence the IL-7 bioactivity. The generation of a modified sCD127 with higher affinity for IL-7 could have therapeutic applications.

An interesting biological compound, which acts as a potent downregulator of IL-7R $\alpha$ signaling is the $15 \mathrm{kDa}$ Tat protein produced by the HIV virus [54]. Tat is secreted by HIV infected cells detectable in the serum of HIV-infected patients or in supernatants from in vitro cultures. Tat enters the cytoplasm of CD8 + T cells and interacts with the cytoplasmic tail of the IL$7 \mathrm{R} \alpha$ causing clustering and downregulation from the cell surface. The internalized IL-7R $\alpha$ is then directed to the proteasome for degradation.

Pharmacological modulation of IL-7R $\alpha$ signaling can be achieved by targeting downstream signaling such as JAK1 and JAK3. Tofacitinib is a potent inhibitor of JAK 1 and JAK 3 and currently approved for the treatment of rheumatoid arthritis (RA), has demonstrated effectiveness in the treatment of psoriasis in phase III clinical trials, and is currently tested as immunomodulator for organ transplantation [55]. Tofacitinib was shown to impair Th1 and Th17 effector function; however, its action is broad and includes all $\gamma_{\mathrm{c}}$-chain cytokines. Other JAK inhibitors under evaluation in clinical trials for autoimmune diseases include the JAK1 and JAK2 inhibitor Ruxolitinib, the JAK1 inhibitor GLPG-0634, and the JAK3 antagonist VX-509. Even though none of these compounds is selective for the IL-7/IL-7R $\alpha$ signaling pathway, they can be used in combination with selective inhibitors of the IL-7R $\alpha$ to potentiate the effectiveness.

\section{Targeting IL-7-Mediated Homeostatic T Cell Proliferation in Type 1 Diabetes and Islet Transplantation}

A humanized IgG1 monoclonal antibody that binds to and inhibit the IL-7R $\alpha$ has been developed by GlaxoSmithKline and tested as single ascending doses study phase I clinical trial on healthy volunteers to determine the safety, tolerability, pharmacokinetics, pharmacodynamics, and immunogenicity (NCT02293161, ClinicalTrials.gov identifier). The primary outcome measures were expected in September 2015 and not yet released.

The anti-IL-7R $\alpha$ monoclonal antibody PF-06342674 (RN168) developed from Pfizer has been tested in phase $1 \mathrm{~b}$ in patients with multiple sclerosis (NCT02045732 ClinicalTrials.gov identifier) with the primary outcome to evaluate adverse side effects and pharmacokinetic- 
pharmacodynamic properties. The trial is reported to have been terminated in April 2015 due to a corporate decision not related to safety or tolerability issues. A second trial was designed to test the same antibody in patients with type 1 diabetes and is estimated to conclude in March 2016.

While waiting for the results of these trials in terms of safety and tolerability and for the design of phase II and phase III trials to determine whether blockade of the IL-7R $\alpha$ is effective in the treatment of type 1 diabetes, it is important to highlight the interest and the effort to explore the IL-7/IL-7R axis as a potential target pathway to control beta-cell autoimmunity.

As we previously reported, islet transplantation in patients with type 1 diabetes is associated with IL-7-mediated homeostatic proliferation of memory autoreactive T cells. This model also provided important evidences of the effect of classical immunosuppressive drugs on the IL-7/IL-7R axis. The mTOR inhibitor rapamycin and the calcineurin inhibitor FK506 were ineffective on IL-7-mediated homeostatic T cell proliferation at therapeutic serum level concentrations [14••]. Interestingly, the anti-CD25 antibody daclizumab by blocking the interaction of CD25 with the $\gamma_{\mathrm{c}}$ chain, promotes the association of the IL-7R $\alpha / \gamma_{\mathrm{c}}$ complex resulting in an increased sensitivity of T cells to IL-7 [56]. On the other hand, mycophenolate mofetil (MMF) was shown to reduce by $90 \%$ the rate of homeostatic $\mathrm{T}$ cell proliferation at therapeutic serum level concentrations [14••]. MMF is a purine synthesis inhibitor acting very downstream in the proliferation process. Even though the mechanisms of action suggest that inhibition of $\mathrm{T}$ cell proliferation is not specific it represents the best therapeutic option to control homeostatic proliferation at least in the transplantation setting.

\section{Future Perspectives}

Recent findings suggest that the immune response can be manipulated by targeting the bio-energetic metabolism of T cells. Both naive and memory $\mathrm{T}$ cell subsets are quiescent cells and rely on oxidative phosphorylation to sustain housekeeping functions [57]. However, proliferating cells like activated and effector $\mathrm{T}$ cells upregulate oxidative phosphorylation to meet the increased energetic demand and activates aerobic glycolysis, which is better suited for the biosynthesis of cell components, proliferation, and effector functions [58]. Bz-423 is a novel small molecule that inhibits the mitochondrial F1F0-ATPase resulting in the increase of superoxide and inducing selective apoptosis of alloreactive T cells while sparing proliferating hematopoietic stem cells using preferentially glycolysis [59•]. This strategy was successful in preventing graft vs host disease while preserving immune system repopulation in a mouse model. In a mouse model of systemic lupus erythematosus (SLE), a combination treatment with the glycolysis inhibitor 2-deoxy-D-glucose and the fatty acid oxidation inhibitor metformin was able to normalize bioenergetic metabolism of T cells and reverse SLE [60•]. As previously reported, there is a strong link between IL-7 signaling and bio-energetic metabolism, especially the IL-7 dependent activation of the glycolysis machinery. The T cell bioenergetic metabolism is a novel and quickly developing frontier for developing innovative approaches to control IL-7mediated homeostatic $\mathrm{T}$ cell proliferation and beta-cell autoimmunity.

\section{Conclusions}

IL-7-mediated homeostatic proliferation T cell clones have gained increasing interest in the pathogenesis of type 1 diabetes and in autoimmunity recurrence post pancreas or islet transplantation. This prompted efforts into a better understanding of the molecules and pathways involved in the IL7/IL-7R axis signaling that can be selectively targeted to control homeostatic proliferation. Some of these are in first phase clinical trials, and we need to consider other ways in which homeostatic proliferation can be controlled and incorporate such strategies in the therapy of beta-cell autoimmunity.

Acknowledgments Paolo Monti is supported by a Career Development Award from the Juvenile Diabetes Research Foundation (JDRF, 5-CDA2015-85-A-N).

\section{Compliance with Ethical Standards}

Conflict of Interest Debora Vignali and Paolo Monti declare that they have no conflict of interest.

Human and Animal Rights and Informed Consent This article does not contain any studies with human or animal subjects performed by any of the authors.

Open Access This article is distributed under the terms of the Creative Commons Attribution 4.0 International License (http:// creativecommons.org/licenses/by/4.0/), which permits unrestricted use, distribution, and reproduction in any medium, provided you give appropriate credit to the original author(s) and the source, provide a link to the Creative Commons license, and indicate if changes were made.

\section{References}

Papers of particular interest, published recently, have been highlighted as:

- Of importance

•- Of major importance

1. Roep BO. The role of T-cells in the pathogenesis of Type 1 diabetes: from cause to cure. Diabetologia. 2003;46:305-21. 
2. Di Lorenzo TP, Peakman M, Roep BO. Translational mini-review series on type 1 diabetes: systematic analysis of T cell epitopes in autoimmune diabetes. Clin Exp Immunol. 2007;148:1-16.

3. Mannering SI, Wong FS, Durinovic-Belló I, et al. Current approaches to measuring human islet-antigen specific $\mathrm{T}$ cell function in type 1 diabetes. Clin Exp Immunol. 2010;162:197-209.

4. Danke N, Yang J, Greenbaum C, Kwok WW. Comparative study of GAD65-specific CD4+ T cells in healthy and type 1 diabetic subjects. J Autoimmun. 2005;25:303-11. This paper revealed the presence of GAD65 reactive T cells in subjects with no other sign of autoimmunity.

5. Monti P, Scirpoli M, Rigamonti A, et al. Evidence for in vivo primed and expanded autoreactive $\mathrm{T}$ cells as a specific feature of patients with type 1 diabetes. J Immunol. 2007;179:5785-92. This paper showed that autoreactive $T$ cells in patients with type 1 diabetes developed a memory phenotype.

6. Viglietta V, Kent SC, Orban T, Hafler DA. GAD65-reactive T cells are activated in patients with autoimmune type 1a diabetes. J Clin Invest. 2002;109:895-903.

7. Wherrett DK, Bundy B, Becker DJ, et al. Antigen-based therapy with glutamic acid decarboxylase (GAD) vaccine in patients with recent-onset type 1 diabetes: a randomised double-blind trial. Lancet. 2011;378:319-27.

8. Orban T, Bundy B, Becker DJ, DiMeglio L, et al. Co-stimulation modulation with abatacept in patients with recent-onset type 1 diabetes: a randomised, double-blind, placebo-controlled trial. Lancet. 2011;378:412-9.

9. Sherry N, Hagopian W, Ludvigsson J, et al. Teplizumab for treatment of type 1 diabetes (Protégé study): 1-year results from a randomised, placebo-controlled trial. Lancet. 2011;378:487-97.

10. Voltarelli JC, Couri CEB, Stracieri ABPL, et al. Autologous nonmyeloablative hematopoietic stem cell transplantation in newly diagnosed type 1 diabetes mellitus. JAMA. 2007;297: 1568-76.

11. Couri CEB, de Oliveira MC, Simões BP. Risks, benefits, and therapeutic potential of hematopoietic stem cell transplantation for autoimmune diabetes. Curr Diabet Rep. 2012;12:604-11.

12. Couri CEB, Oliveira MCB, Stracieri ABPL, et al. C-peptide levels and insulin independence following autologous nonmyeloablative hematopoietic stem cell transplantation in newly diagnosed type 1 diabetes mellitus. JAMA. 2009;301(15):1573-9.

13. Monti P, Bonifacio E. Interleukin-7 and type 1 diabetes. Curr Diabet Rep. 2014;14:518-26.

14.• Monti P, Scirpoli M, Maffi P, Ghidoli N, De TF, Bertuzzi F, et al. Islet transplantation in patients with autoimmune diabetes induces homeostatic cytokines that expand autoreactive memory $\mathrm{T}$ cells. $\mathrm{J}$ Clin Invest. 2008;118:1806-14. This study reported that lymphopenia is associated with increased circulating IL-7 which drives expansion of autoreactive $T$ cell clones in patients with type 1 diabetes undergoing to islet allo-transplantation.

15.• Cieri N, Camisa B, Cocchiarella F, Forcato M, Oliveira G, Provasi E, et al. IL-7 and IL-15 instruct the generation of human memory stem T cells from naive precursors. Blood. 2013;121:573-84. This study demonstrated that homeostatic cytokines IL-7 and IL-15 are involved in the generation of memory stem $T$ cells.

16. Chehtane M, Khaled AR. Interleukin-7 mediates glucose utilization in lymphocytes through transcriptional regulation of the hexokinase II gene. Am J Physiol Cell Physiol. 2010;298:1560-71.

17. Mazzucchelli RI, Warming S, Lawrence SM, Ishii M, Abshari M, Washington AV, et al. Visualization and identification of IL-7 producing cells in reporter mice. PLoS ONE. 2009;4:e7637.

18. Mazzucchelli R, Durum SK. Interleukin-7 receptor expression: intelligent design. Nat Rev Immunol. 2007;7(2):144-54.

19. Kovanen PE, Leonard WJ. Cytokines and immunodeficiency diseases: critical roles of the $\gamma$ c-dependent cytokines interleukins 2,4 ,
$7,9,15$, and 21 , and their signaling pathways. Immunol Rev. 2004;202:67-83.

20. Maraskovsky E, O'Reilly LA, Teepe M, et al. Bcl-2 can rescue T lymphocyte development in interleukin-7 receptor-deficient mice but not in mutant rag-1-/- mice. Cell. 1997;89:1011-9.

21. Pellegrini M, Bouillet $\mathrm{P}$, Robati M, et al. Loss of bim increases $\mathrm{T}$ cell production and function in interleukin 7 receptor-deficient mice. J Exp Med. 2004;200:1189-95.

22. Li WQ, Jiang Q, Aleem E, et al. IL-7 promotes T cell proliferation through destabilization of p27Kip1. J Exp Med. 2006;203:573-82.

23. Khaled AR, Bulavin DV, Kittipatarin C, et al. Cytokine-driven cell cycling is mediated through Cdc25A. J Cell Biol. 2005;169:755-63.

24. Fox CJ, Hammerman PS, Thompson CB. Fuel feeds function: energy metabolism and the T-cell response. Nat Rev Immunol. 2005;5:844-52.

25. Pearce EL. Metabolism in T cell activation and differentiation. Curr Opin Immunol. 2010;22:314-20.

26. Wofford JA, Wieman HL, Jacobs SR, et al. IL-7 promotes Glut1 trafficking and glucose uptake via STAT5-mediated activation of Akt to support T-cell survival. Blood. 2008;111:2101-11.

27. Rathmell JC, Farkash EA, Gao W, et al. IL-7 enhances the survival and maintains the size of naive T cells. J Immunol. 2001;167:686976.

28. Khoruts A, Fraser JM. A causal link between lymphopenia and autoimmunity. Immunol Lett. 2005;98:23-31.

29. Goldrath AW, Bevan MJ. Low-affinity ligands for the TCR drive proliferation of mature CD8+ T cells in lymphopenic hosts. Immunity. 1999;11:183-90. This study showed the selective advantage a $T$ cells with a low affinity $T$ cell Receptor during homeostatic $T$ cell proliferation.

30. Sallusto F, Geginat J, Lanzavecchia A. Central memory and effector memory $\mathrm{T}$ cell subsets: function, generation, and maintenance. Annu Rev Immunol. 2004;22:745-63.

31. King C, Ilic A, Koelsch K, et al. Homeostatic expansion of T cells during immune insufficiency generates autoimmunity. Cell. 2004;117:265-77.

32. Calzascia T, Pellegrini M, Lin A, et al. CD4 T cells, lymphopenia, and IL-7 in a multistep pathway to autoimmunity. PNAS. 2008;105: 2999-3004. This study directly correlated IL-7 with the development of autoimmune diabetes in a mouse model.

33. Lundmark F, Duvefelt K, Iacobaeus E, et al. Variation in interleukin 7 receptor $\alpha$ chain (IL7R) influences risk of multiple sclerosis. Nat Genet. 2007:39:1108-13.

34. Monti P, Brigatti C, Krasmann M, et al. Concentration and activity of the soluble form of the interleukin-7 receptor alpha in type I diabetes identifies an interplay between hyperglycemia and immune function. Diabetes. 2013;62:2500-8.

35. Heninger A-K, Monti P, Wilhelm C, et al. Activation of islet autoreactive naive $\mathrm{T}$ cells in infants is influenced by homeostatic mechanisms and antigen-presenting capacity. Diabetes. 2013;62: 2059-66.

36. Heninger A-K, Theil A, Wilhelm C, et al. IL-7 abrogates suppressive activity of human $\mathrm{CD} 4+\mathrm{CD} 25+\mathrm{FOXP} 3+$ regulatory $\mathrm{T}$ cells and allows expansion of alloreactive and autoreactive T cells. $\mathrm{J}$ Immunol. 2012;189(12):5649-58. This study reported that high IL-7 concentrations can inhibit the suppressive capacity of CD4 + CD25 + FOXP3 + regulatory $T$ cells.

37. Sallusto F, Lenig D, Förster R, et al. Two subsets of memory T lymphocytes with distinct homing potentials and effector functions. Nature. 1999;401:708-12.

38. Restifo NP, Gattinoni L. Lineage relationship of effector and memory T cells. Curr Opin Immunol. 2013;25:556-63.

39. Macallan DC, Wallace D, Zhang Y, et al. Rapid turnover of effectormemory CD4(+) T cells in healthy humans. J Exp Med. 2004;200: 255-60. 
40. Vrisekoop N, den Braber I, de Boer AB, et al. Sparse production but preferential incorporation of recently produced naive $\mathrm{T}$ cells in the human peripheral pool. Proc Natl Acad Sci U S A. 2008;105:611520 .

41.• Gattinoni L, Zhong X-S, Palmer D, et al. Wnt signaling arrests effector T cell differentiation and generates CD8+ memory stem cells. Nat Med. 2009;15:808-13. This study reported for the first time the generation of memory stem $\mathrm{T}$ cells by interfering with the WNT signaling pathway.

42.• Lugli E, Dominguez MH, Gattinoni L, et al. Brief report Superior $\mathrm{T}$ memory stem cell persistence supports long-lived T cell memory. J Clin Invest. 2013;123:594-9. This study reported for the first time the existence and phenotype of memory stem $T$ cells in humans.

43. Laughlin E, Burke G, Pugliese A. Autoimmune diabetes after pancreas transplantation. Clin Immunol. 2008;128:23-30.

44. Roep BO, Stobbe I, Duinkerken G, et al. Auto- and alloimmune reactivity to human islet allografts transplanted into type 1 diabetic patients. Diabetes. 1999;48:484-90.

45. Monti P, Heninger A-K, Bonifacio E. Differentiation, expansion, and homeostasis of autoreactive T cells in type 1 diabetes mellitus. Curr Diabet Rep. 2009;9:113-8.

46. Beck B, Blanpain C. Unravelling cancer stem cell potential. Nat Rev Cancer. 2013;13:727-38.

47.• Penaranda C, Kuswanto W, Hofmann J, et al. IL-7 receptor blockade reverses autoimmune diabetes by promoting inhibition of effector/memory T cells. PNAS. 2012;109:12668-73. This study and the following reference show reversal of autoimmune diabetes in the NOD mouse model using a monoclonal antibody directed to the IL-7R $\alpha$.

48.• Lee L, Logronio K, Huan G, et al. Anti-IL-7 receptor- $\alpha$ reverses established type 1 diabetes in nonobese diabetic mice by modulating effector T-cell function. PNAS. 2012;109:12674-9. Companion paper of Penaranda et al. ref 45.

49. Le MH, Boeffard F, Longis J, et al. IL-7 receptor blockade following $\mathrm{T}$ cell depletion promotes long-term allograft survival. J Clin Invest. 2014;124:1723-33. This study showed that a monoclonal antibody directed to the IL-7R $\alpha$ induce islet graft acceptance in a non-autoimmune model of diabetes.
50. Goodwin RG, Friend D, Ziegler SF, et al. Cloning of the human and murine interleukin-7 receptors: demonstration of a soluble form and homology to a new receptor superfamily. Cell. 1990;60:941-51.

51. McKay FC, Swain LI, Schibeci SD, et al. Haplotypes of the interleukin 7 receptor alpha gene are correlated with altered expression in whole blood cells in multiple sclerosis. Genes Immunol. 2008;9: $1-6$.

52. Teutsch SM, Booth DR, Bennetts BH, et al. Identification of 11 novel and common single nucleotide polymorphisms in the interleukin-7 receptor-alpha gene and their associations with multiple sclerosis. Eur J Hum Genet. 2003;11:509-15.

53. Noguchi M, Nakamura Y, Russell SM, et al. Interleukin-2 receptor gamma chain: a functional component of the interleukin-7 receptor. Science. 1993;262:1877-80.

54. Faller EM, Sugden SM, McVey MJ, et al. Soluble HIV Tat protein removes the IL-7 receptor alpha-chain from the surface of resting CD8 T cells and targets it for degradation. J Immunol. 2010;185: 2854-66.

55. Tanaka Y. Recent progress and perspective in JAK inhibitors for rheumatoid arthritis: from bench to bedside. J Biochem. 2015;158: $173-9$.

56. Monti P, Brigatti C, Heninger A-K, et al. Disengaging the IL-2 receptor with daclizumab enhances IL-7-mediated proliferation of CD4(+) and CD8(+) T cells. Am J Transplant. 2009;9:2727-35.

57. Jones RG, Thompson CB. Revving the engine: signal transduction fuels T cell activation. Immunity. 2007;27:173-8.

58. Wang R, Green DR. Metabolic checkpoints in activated T cells. Nat Immunol. 2012;13:907-15.

59. Gatza E, Wahl DR, Opipari AW, et al. Manipulating the bioenergetics of alloreactive $T$ cells causes their selective apoptosis and arrests graft versus host disease. Sci Transl Med. 2011;3:1-16. This paper showed that GVDH can be controlled by targeting the mitochondrial f0f1 ATP-ase.

60. Yin Y, Choi SS-C, Xu Z, Perry DJ, Seay H, Croker BP, et al. Normalization of CD4+ T cell metabolism reverses lupus. Sci Transl Med. 2015;7:274ra18. This paper showed that a combination of the glycolysis inhibitor 2-DG and the oxidative phosphorylation inhibitor metformin can stop lupus in a mouse model. 